\title{
Hypertension as a Predictive Factor for Survival Outcomes in Patients with Metastatic Renal Cell Carcinoma Treated with Sunitinib after Progression on Cytokines
}

\author{
Sebastian Szmit ${ }^{a}$ b Przemysław Langiewicz ${ }^{a} \quad$ Jakub Żołnierek $^{a}$ \\ Paweł Nurzyński ${ }^{a}$ Magdalena Zaborowska ${ }^{a}$ Krzysztof J. Filipiak ${ }^{b}$ \\ Grzegorz Opolski ${ }^{b}$ Cezary Szczylik ${ }^{a}$
}

${ }^{a}$ Department of Oncology, Military Institute of Medicine, and ${ }^{b}$ First Department of Cardiology, Medical University of

Warsaw, Warsaw, Poland

\section{Key Words}

Hypertension • Renal cell carcinoma • Sunitinib •

Antihypertensive treatment

\begin{abstract}
Background/Aims: This retrospective analysis compared progression-free survival (PFS) in 111 patients who developed or had preexisting hypertension with those who did not during treatment with second-line sunitinib. Secondary objectives included overall survival (OS) and safety. Methods: Patients with metastatic renal cell carcinoma ( $\mathrm{mRCC}$ ) received sunitinib $50 \mathrm{mg}$ orally once daily in 6-week cycles according to a 4-week on/2-week off treatment schedule. Treatment was continued until disease progression, unacceptable toxicity, withdrawal of consent, or death. Resting blood pressure (BP) was monitored by clinic and home measurements. Hypertension was defined as systolic BP $\geq 140$ and/or diastolic BP $\geq 90 \mathrm{~mm} \mathrm{Hg}$. Subsequent antihypertensive treatment was empirical, depending on the patient. Results: Fifty-four (48.6\%) patients experienced elevated $\mathrm{BP}$ related to sunitinib. Of these, 10 had preexisting hypertension. Patients who developed hypertension related to sunitinib treatment experienced significantly longer PFS and OS compared to those who did not ( $p<0.00001)$. Patients
\end{abstract}

who required at least 3 antihypertensive drugs had the longest PFS ( $p=0.00002)$ and OS ( $p=0.00001)$. Conclusions: The development of hypertension during sunitinib treatment was a positive predictive factor associated with a significantly longer PFS and OS in patients with $\mathrm{mRCC}$.

Copyright $\odot 2011$ S. Karger AG, Basel

\section{Introduction}

Hypertension is a known class effect of targeted antiangiogenic therapy, the current mainstay of treatment for several solid tumor cancers $[1,2]$. Systemic inhibition of vascular endothelial growth factor (VEGF) receptor signaling reduces capillary density, which appears to provoke hypertension and may eventually lead to contractile dysfunction or heart failure [3, 4]. Similarly, the mechanism of action by which VEGF inhibition induces hypertension is not well established. It has been postulated, however, that VEGF stimulation leads to activation of nitric oxide and prostacyclin, both known vasodilators, and thus VEGF inhibition could lead to decreased activity of these vasodilators [5]. Sunitinib malate (SUTENT ${ }^{\circledR}$; Pfizer Inc.) is a potent multitargeted inhibitor of VEGF receptor 1-3 and PDGFR (platelet-derived growth factor

\section{KARGER \\ Fax +41613061234 E-Mail karger@karger.ch} www.karger.com

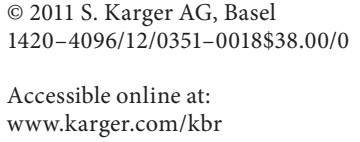

\footnotetext{
Sebastian Szmit

Department of Oncology, Military Institute of Medicine

Szaserow Street 128

PL-00-909 Warsaw (Poland)

Tel. +48 22610 3098, E-Mail s.szmit@gmail.com
} 
receptor) and currently approved worldwide for first-line treatment of patients with metastatic renal cell carcinoma (mRCC) (Pfizer Inc., 2008). In line with its drug class, there is some evidence that sunitinib therapy induces hypertension and decreases left-ventricular ejection fraction in patients with mRCC [7]. However, sunitinib-related myocardial dysfunction may be completely reversible with suitable cardioprotective treatment [8].

According to a meta-analysis performed by Zhu and coworkers in 2009, the overall frequency of hypertension (all degrees) during sunitinib therapy in published clinical studies was $21.6 \%$ (95\% CI 18.7-24.8\%) and the incidence of third- or fourth-degree hypertension was $6.8 \%$ (95\% CI 5.3-8.8\%). The risk of developing hypertension seemed to vary with tumor type and the dosing schedule with sunitinib [9]. Further, it has been suggested that the development of hypertension during treatment with VEGF inhibitors could be a biomarker of antitumor efficacy $[9,10]$. In an analysis of the association between sunitinib-induced hypertension and clinical outcome in mRCC patients from four prospective clinical trials, hypertension was a significantly positive independent predictor of progression-free survival (PFS) and overall survival (OS) [9]. Antihypertensive medication use did not affect clinical outcome in this analysis. Current evidence surrounding hypertension and heart failure as sequential side effects of sunitinib is limited. While the cardiologic care of patients who develop hypertension during treatment with sunitinib is crucial, optimal treatment plans remain to be elucidated [11]. This study was initiated to further investigate the optimal management of patients developing hypertension while receiving sunitinib and the association between arterial hypertension and survival outcomes in patients with mRCC who develop hypertension during treatment with sunitinib.

\section{Subjects and Methods}

\section{Patients}

Patients aged 18 years or older, with histologically proven clear cell mRCC who had undergone previous nephrectomy and were classified as intermediate- or low-risk patients, according to $\mathrm{Me}$ morial Sloan-Kettering Cancer Center (MSKCC) criteria, were included in the study [12]. All patients had received cytokine immunotherapy previously; sunitinib was not approved in Poland for first-line treatment of mRCC until 2009. All patients had adequate organ function as defined by the following: total serum bilirubin $\leq 2 \times$ the upper limit of normal (ULN), serum transaminases $<5 \times$ ULN, serum creatinine $\leq 2 \times \mathrm{ULN}$, absolute neutrophil count $\geq 1,000 / \mathrm{mm}^{3}$ without growth factor support, platelets $\geq 75,000 / \mathrm{mm}^{3}$, and hemoglobin $\geq 8.0 \mathrm{~g} / \mathrm{dl}$. Required
ECOG (Eastern Cooperative Oncology Group) performance status was 0,1 or 2. Patients provided written informed consent.

Exclusion criteria included congestive heart failure, myocardial infarction or coronary artery bypass graft in the previous 6 months, ongoing severe or unstable angina, or any unstable arrhythmia requiring medication. Preexisting hypertension was not a contraindication for treatment with sunitinib, but the authors decided that all patients had to control BP below 140/90 mm Hg.

\section{Study Design and Treatment}

This retrospective cohort study included all patients treated for mRCC between January 1, 2005 and July 30, 2009 at the Department of Oncology at the Military Institute of Medicine in Warsaw, Poland. Patients with mRCC received sunitinib $50 \mathrm{mg}$ orally once daily in repeated 6 -week cycles according to a $4 / 2$ schedule (4 weeks on treatment followed by 2 weeks without treatment). Patients were monitored closely for toxicity and dose reductions were allowed depending on the type and severity of toxicity. For treatment-related hypertension, treatment would be stopped for patients experiencing grade 4 hypertension (according to Common Terminology Criteria for Adverse Events, CTCAE, v3.0). In instances of less than grade 4 hypertension, dose reductions of sunitinib were recommended for patients whose blood pressure (BP) was difficult to control. This study was approved by the local Ethics Committee.

Control visits were planned as follows: cycle 1: day 1, day 14, day 28; cycle 2: day 1 and day 28; cycle 3 and all following cycles: day 1 . Treatment with sunitinib was continued until disease progression, unacceptable toxicity, withdrawal of consent, or death occurred. Disease progression was assessed using Response Evaluation Criteria in Solid Tumors (RECIST) [13].

The study complied with the provisions of the Declaration of Helsinki, Good Clinical Practice Guidelines, and local laws and regulations.

\section{Efficacy and Safety Assessments}

The primary objective of this study was to compare PFS between patients who developed hypertension with those who did not develop hypertension during the first cycle of treatment with sunitinib. PFS was defined as the time from the start of sunitinib therapy until disease progression or death from any cause. Secondary objectives included OS between hypertensive and normotensive patients, safety, and a comparative analysis of the efficacy of antihypertensive treatments in relation to survival. OS was defined as the time from the start of sunitinib therapy to death. Heart failure was assessed according to the Boston criteria [14].

Safety was assessed at regular intervals by documentation of adverse events and physical examination. Laboratory assessments (hematologic and serum chemical measurements) were performed throughout the study by a central laboratory. Adverse events were graded using the National Cancer Institute (NCI) CTCAE version 3.0.

Disease progression was measured with CT scan by independent radiologists. Every patient was assessed every 3 cycles (18 weeks).

Diagnosis and Treatment of Hypertension

All patients were required to measure their BP three times a day at rest during active treatment with sunitinib and during the 2 weeks off treatment. Home BP monitoring was compatible with 
Table 1. Baseline characteristics of patients who developed hypertension related to sunitinib and who remained normotensive throughout the study

\begin{tabular}{|c|c|c|}
\hline Characteristic & Hypertensive group $(\mathrm{n}=54)$ & Normotensive group $(n=57)$ \\
\hline Mean age \pm SD (range), years & $56.6 \pm 10.4(26-75)$ & $55.2 \pm 9.4(26-73)$ \\
\hline Gender (M/F), n & $41 / 13$ & $42 / 15$ \\
\hline Preexisting hypertension, $\mathrm{n}(\%)$ & $10(18.5)$ & $20(35.1)$ \\
\hline \multicolumn{3}{|l|}{ Metastatic sites, $\mathrm{n}(\%)$} \\
\hline Lung & $39(72.2)$ & $40(70.2)$ \\
\hline Liver & $17(31.5)$ & $23(40.4)$ \\
\hline Lymph nodes & $31(57.4)$ & $29(50.9)$ \\
\hline Local recurrence & $17(31.5)$ & $18(31.6)$ \\
\hline Bone & $14(25.9)$ & $18(31.6)$ \\
\hline Central nervous system & $3(5.6)$ & $6(10.5)$ \\
\hline \multicolumn{3}{|l|}{ MSKCC risk group, n (\%) } \\
\hline Intermediate & $45(83.3)$ & $55(96.5)$ \\
\hline Low & $9(16.6)$ & $2(3.5)$ \\
\hline \multicolumn{3}{|l|}{ ECOG PS, n (\%) } \\
\hline 0 & $27(50.0)$ & $19(33.3)$ \\
\hline 1 & $24(44.4)$ & $33(57.9)$ \\
\hline 2 & $3(5.6)$ & $5(8.8)$ \\
\hline \multicolumn{3}{|l|}{ Mean $\mathrm{BP} \pm \mathrm{SD}, \mathrm{mm} \mathrm{Hg}$} \\
\hline \multicolumn{3}{|l|}{ At baseline } \\
\hline Systolic & $125.7 \pm 7.1$ & $124.2 \pm 8.9$ \\
\hline Diastolic & $74.2 \pm 6.0$ & $75.2 \pm 5.9$ \\
\hline \multicolumn{3}{|c|}{$\begin{array}{l}\text { Mean values of } 28 \text { days of first cycle } \\
\text { of sunitinib therapy* }\end{array}$} \\
\hline Systolic & $149.6 \pm 7.4$ & $121.5 \pm 10.6$ \\
\hline Diastolic & $94.1 \pm 7.3$ & $74.8 \pm 6.3$ \\
\hline
\end{tabular}

ECOG $=$ Eastern Cooperative Oncology Group; MSKCC = Memorial Sloan-Kettering Cancer Center; PS = performance status. ${ }^{*} \mathrm{p}<0.0001$.

the most current guidelines from the European Hypertension Society (EHS) in place during the time of the study $[15,16]$. During the clinic visit, $\mathrm{BP}$ was measured twice by the nurses in the clinic, and, additionally, patients presented their home BP measurements. If two independent clinic measurements and the mean value of home systolic and/or diastolic BP measurements were $\geq 140$ and/or $\geq 90 \mathrm{~mm} \mathrm{Hg}$, respectively, this was recognized as hypertension associated with sunitinib therapy. This algorithm gave a confident diagnosis of iatrogenic hypertension. However patients were included in the "hypertensive group" for study analyses only if they had two independent clinic measurements and a mean value of home systolic and/or diastolic BP measurements $\geq 140$ and/or $\geq 90 \mathrm{~mm} \mathrm{Hg}$, respectively, during the 28 days of the first treatment cycle with sunitinib and prior to the initiation of antihypertensive therapy.

Antihypertensive treatment was empirical, in line with obligatory guidelines and depended on the individual clinical situation of each particular patient. Conventional antihypertensive therapies were used to treat sunitinib-induced hypertension including angiotensin-converting enzyme inhibitors (ACEI: enalapril, captopril, ramipril, or lisinopril), $\beta$-blockers (metoprolol, bisoprolol, carvedilol, betaxolol, or atenolol), calcium-channel blockers (amlodipine), thiazide diuretics (hydrochlorothiazide or indapamide) and $\alpha$-blockers (doxazosin). All efforts were made to control BP below 140/90 mm Hg (clinic measurements) and below 135/85 $\mathrm{mm} \mathrm{Hg}$ (home monitoring).

\section{Statistical Methods}

All statistical analyses were performed using STATISTICA 7.0 software. Comparisons between and within groups ('hypertensive' and 'normotensive') were performed using Student's t test or $\chi^{2}$ tests (verified for statistical significance with the Yates test) at a 5\% significance level. OS and PFS were generated with the $\mathrm{Ka}-$ plan-Meier survival analysis method and compared using a logrank test.

\section{Results}

\section{Patient Characteristics}

Overall, 111 patients ( 83 men, 28 women) with mRCC were enrolled in the study. The baseline characteristics of patients with and without hypertension during treatment with sunitinib are shown in table 1 . Overall me- 


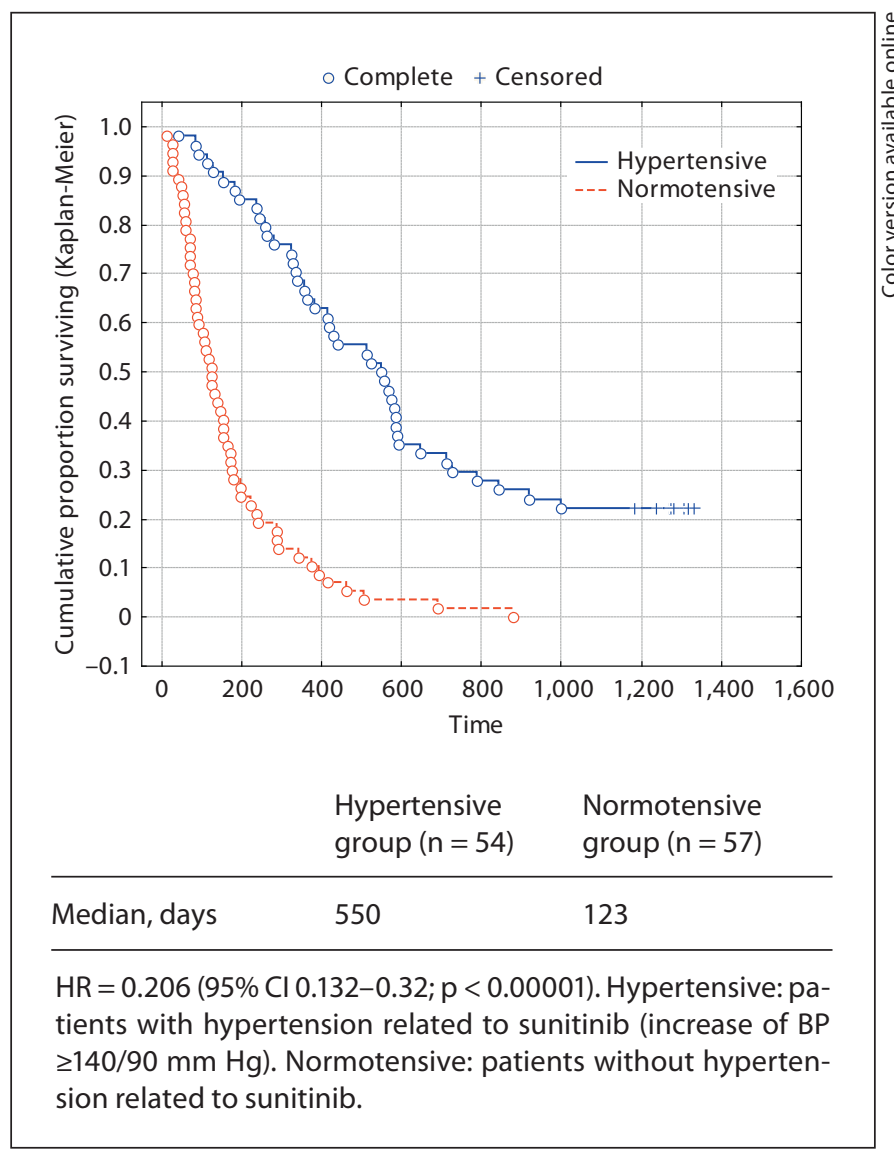

Fig. 1. PFS in mRCC patients who did (hypertensive) or did not (normotensive) develop hypertension when treated with sunitinib.

dian age at the beginning of treatment was 56 years. Preexisting hypertension occurred more frequently in the normotensive group. Thirty patients had preexisting hypertension, which was controlled at baseline to $<130 / 80 \mathrm{~mm} \mathrm{Hg}$ based on household measurements. No patients had recognized organic heart disease or cardiomyopathy (echocardiography examination), symptoms of coronary heart disease, heart failure, or heart rhythm disturbances.

A total of 99 patients (89.2\%) completed therapy with sunitinib, 12 were alive and continued sunitinib therapy after the end of follow-up. The main cause of death was progressive disease and its immediate complications (respiratory failure, cachexia, kidney or liver dysfunction). There was one episode of cardiac tamponade due to disease progression and 2 cases of pulmonary embolism. There were no cardiac deaths related to treatment with sunitinib.

Hypertension and Survival Outcomes in Patients Receiving Sunitinib

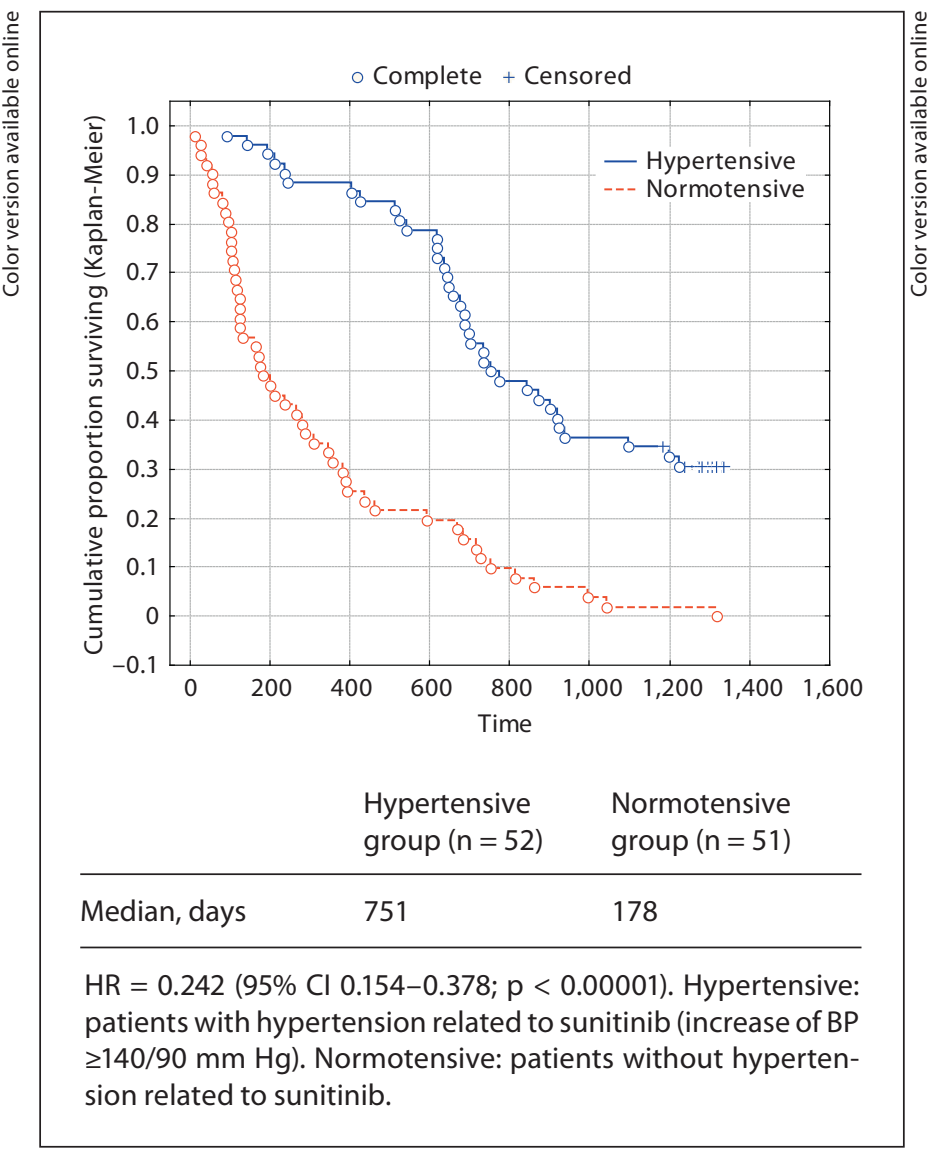

Fig. 2. OS in mRCC patients who did (hypertensive) or did not (normotensive) develop hypertension when treated with sunitinib.

\section{Development of Hypertension}

Overall, 54 (48.6\%) patients developed hypertension during treatment with sunitinib (hypertensive group). Of these patients, 44 (81.5\%) did not have a history of hypertension at baseline, while $10(18.5 \%)$ had preexisting hypertension at baseline. Another 20 patients had preexisting hypertension at baseline but did not develop hypertension during treatment. All patients were diagnosed with iatrogenic hypertension during the 28 days of the first cycle of sunitinib therapy. No patients experienced an increase of BP above 200/110 mm Hg. Antihypertensive treatment was used or modified after the first or second cycle of sunitinib. All patients who received antihypertensive treatment reached the BP goal of $<140 / 90 \mathrm{~mm} \mathrm{Hg}$ (clinic measurements) and $<135 / 85 \mathrm{~mm}$ $\mathrm{Hg}$ (home monitoring), and hypertension did not result in any interruption of the therapy or dose reductions of sunitinib.

Kidney Blood Press Res 2012;35:18-25 


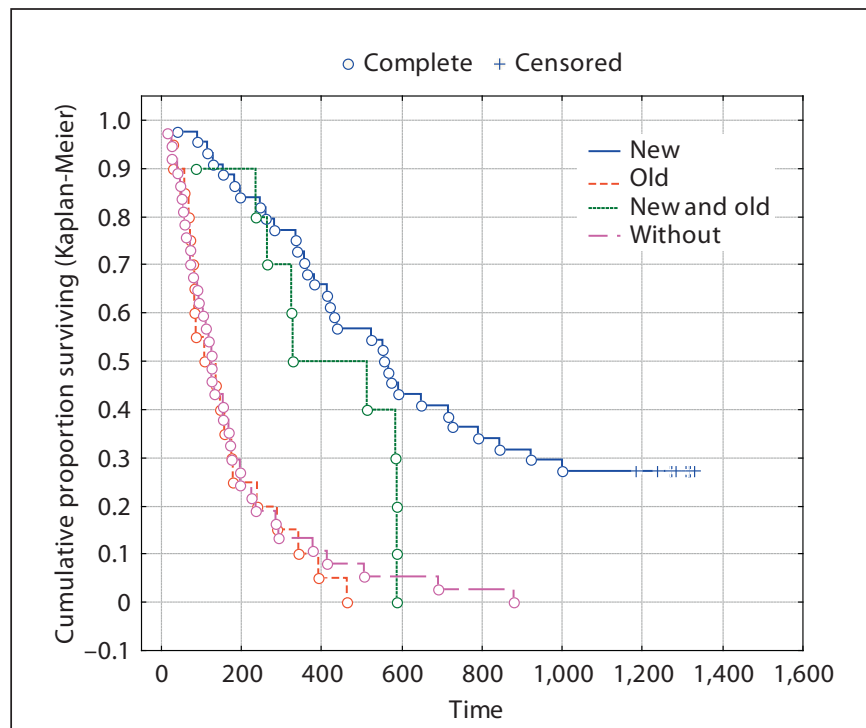

\begin{tabular}{lllll} 
& $\begin{array}{l}\text { Hypertensive group } \\
(n=54)\end{array}$ & & $\begin{array}{l}\text { Normotensive group } \\
(n=57)\end{array}$ \\
\cline { 2 - 3 } $\begin{array}{l}\text { new } \\
(n=44)\end{array}$ & $\begin{array}{l}\text { new and old } \\
(n=10)\end{array}$ & & $\begin{array}{l}\text { old } \\
(n=20)\end{array}$ & $\begin{array}{l}\text { without } \\
(n=37)\end{array}$ \\
\hline $\begin{array}{l}\text { Median } \\
\text { days }\end{array}$ & 556 & 327 & 105 & 123 \\
& $\begin{array}{l}\text { no significant difference } \\
(p>0.05)\end{array}$ & \multicolumn{2}{l}{$\begin{array}{l}\text { no significant difference } \\
(p>0.05)\end{array}$}
\end{tabular}

Hypertensive new: patients who were normotensive before treatment with sunitinib but developed hypertension on treatment. Hypertensive new and old: patients who had preexisting hypertension and developed hypertension while on treatment with sunitinib. Normotensive old: patients with preexisting hypertension who were normotensive during treatment with sunitinib. Normotensive without: patients who were normotensive prior to sunitinib and remained normotensive during treatment with sunitinib.

Fig. 3. PFS in mRCC patients: subgroup analysis dependent on whether the patients had preexisting hypertension $(\mathrm{p}<0.00001)$.

\section{Progression-Free and Overall Survival}

PFS was analyzed for the entire population of 111 patients. Patients with radiological disease progression stopped sunitinib and were offered the next possible oncological treatment, most often sorafenib or everolimus. OS analysis was performed in 103 patients because 8 patients were lost to follow-up (they did not come to clinic visits and could not be reached by telephone).

In the PFS analysis there were 12 patients with a censored date of treatment - they were alive without disease

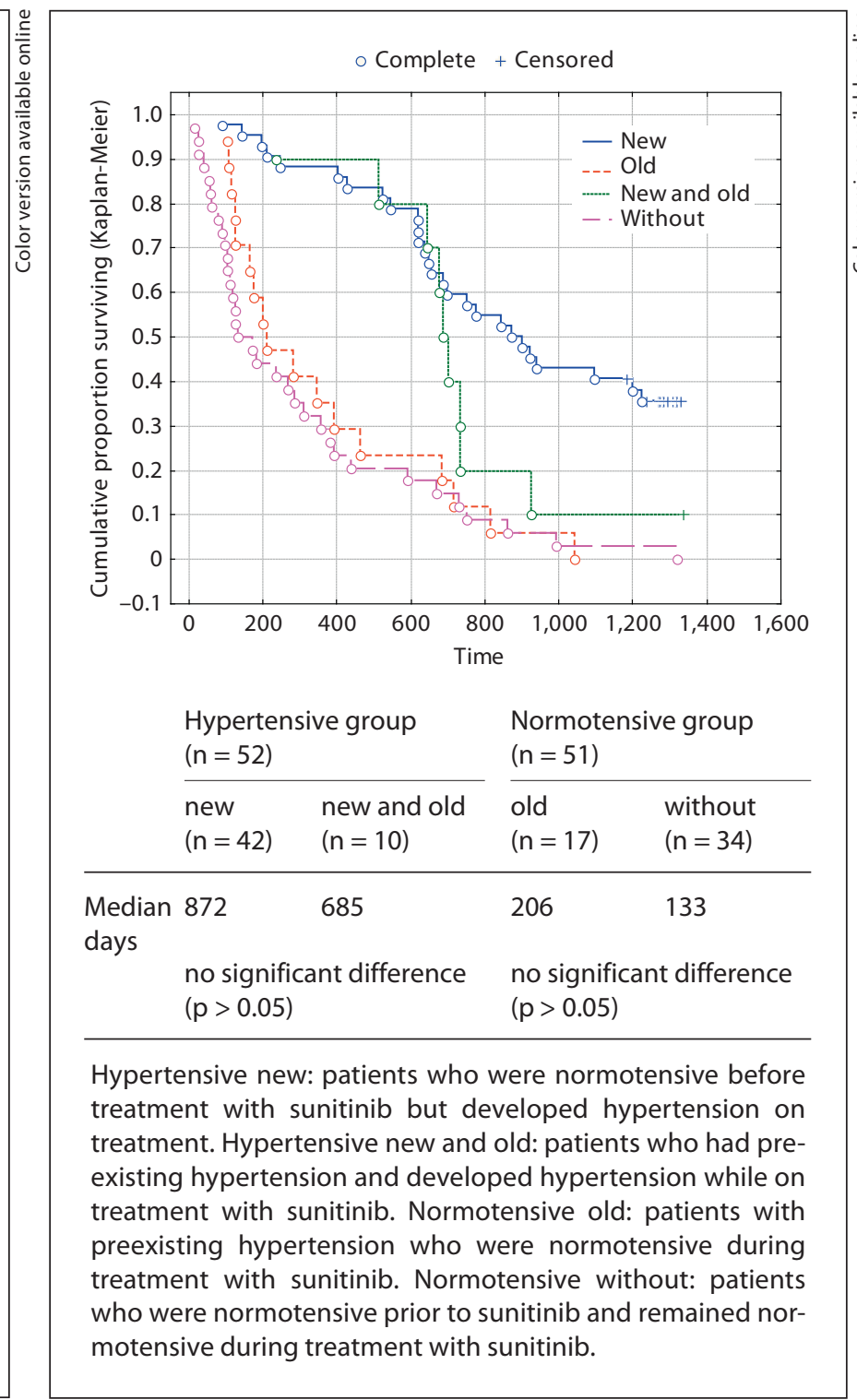

Fig. 4. OS in mRCC patients: subgroup analysis dependent on whether the patients had preexisting hypertension $(\mathrm{p}<0.00001)$.

progression and continued the sunitinib therapy after the end of follow-up.

Patients in the hypertensive group experienced significantly longer PFS (550 vs. 123 days; $p<0.00001$ ) and OS (751 vs. 178 days; $\mathrm{p}<0.00001$ ) compared with normotensive patients during the course of the study (fig. 1, 2). Patients with iatrogenic hypertension had nearly five times lower risk of disease progression $(\mathrm{HR}=0.206 ; 95 \% \mathrm{CI}$ $0.132-0.32$ ) and nearly four times lower risk of mortality $(\mathrm{HR}=0.242$; 95\% CI 0.154-0.378). Further, the subgroup 
of patients with a history of optimally treated hypertension who experienced BP $\geq 140 / 90 \mathrm{~mm} \mathrm{Hg}$ during sunitinib treatment also experienced significantly longer PFS and OS (fig. 3,4) compared with normotensive patients (both $\mathrm{p}<0.00001)$. Moreover, in patients without changes in $\mathrm{BP}$ related to sunitinib, those patients with preexisting hypertension had similar PFS and OS in comparison to patients without preexisting hypertension.

\section{Cardiac Adverse Events}

Symptoms of heart failure appeared in 1 patient with hypertension related to sunitinib. This patient experienced resting dyspnea and leg edema. Echocardiography revealed a coexisting decrease of left-ventricular ejection fraction below $45 \%$ and possible metastases in the myocardium; a chest X-ray showed mild-passive congestion in the lungs. The patient had simultaneous failure of the remaining kidney; he required dialysis and died few days later. The substantial cause of death was disease progression.

An additional patient with hypertension related to sunitinib but without preexisting hypertension experienced asymptomatic atrial fibrillation. He continued sunitinib treatment without dose modification but then ended treatment due to disease progression. One patient with pre-existing hypertension (not related to sunitinib) had recognized atrial fibrillation during sunitinib treatment. He did not require dose modification and ended treatment due to death related to disease progression. Finally, one normotensive man experienced cardiac tamponade. The direct reason of this complication was disease progression with pericardial effusion and he died due to this complication.

\section{Antihypertensive Management}

Of the total 111 patients, 54 developed hypertension during treatment with sunitinib. Of patients with preexisting or iatrogenic hypertension, 19 patients received one antihypertensive medication, 21 received two drugs, 22 received three drugs, and 11 received four drugs. One patient required therapy with five drugs.

Of the 44 patients who developed hypertension related to sunitinib (and without preexisting hypertension), 9 received one antihypertensive medicine, 12 received two drugs, 14 received three drugs, and 8 received four antihypertensive drugs. One patient required therapy with five drugs.

The most frequently used medicines in every specified group were calcium antagonists, $\beta$-blockers, ACEIs, and thiazide diuretics. Notably, patients treated with at least three antihypertensive drugs experienced significantly longer PFS $(\mathrm{p}=0.00002)$ and OS $(\mathrm{p}=0.00001)$ compared either with patients who received one or two medications or with patients who received no medications.

\section{Discussion}

In this retrospective study, a clear association between the development of hypertension during treatment with sunitinib and survival outcome of patients with mRCC has been established. Overall, the results indicate that a sunitinib-related increase in BP above $140 / 90 \mathrm{~mm} \mathrm{Hg}$ is a potentially positive prognostic factor for survival in patients with mRCC, and is associated with both a significantly lower risk of total mortality and significantly longer PFS. Indeed, our findings may suggest that the development of grade 3 hypertension (CTCAE) during treatment with sunitinib is associated with a good oncological prognosis. Rini et al. [10] were the first to describe sunitinib-induced hypertension as a strong positive predictive value for PFS and OS in mRCC patients. Recently, Bono et al. [17] found similar results in a retrospective trial of 64 consecutive mRCC patients treated with sunitinib. In this trial, $38 \%$ of patients developed hypertension during treatment with sunitinib and were found to have significant increases in overall response rate, PFS and OS in comparison to patients who did not develop hypertension. Our study results further confirm those of Rini et al. [10] and Bono et al. [17] and extend the observation to specifically include subsets of patients with controlled hypertension and those normotensive prior to sunitinib therapy. The results are also in agreement with previous analyses showing an association of hypertension induced by bevacizumab with increased PFS [18] and by axitinib with increased OS [9]. Furthermore, Sire et al. [19] have shown that the development of second-degree (or higher) hypertension is a positive prognostic factor for patients with mRCC treated with first- or second-line sunitinib, sorafenib, or bevacizumab. Nevertheless, BP as a potential efficacy marker requires validation in welldesigned prospective clinical trials.

Although uncontrolled high BP is associated with an increased risk of developing $\mathrm{mRCC}$ [20], it is also a contraindication for treatment with sunitinib. A multifactorial analysis performed by Di Lorenzo et al. [21] demonstrated that a history of coronary heart disease and hypertension were strong risk factors for heart failure in patients with mRCC treated with sunitinib. We did not observe such a correlation in our study. Preexisting hy- 
pertension did not worsen the prognosis of patients with mRCC in this study and preexisting and iatrogenic hypertension did not correlate with clinically significant cardiologic events. This is in agreement with Schmidinger et al. [22] who demonstrated that patients with symptoms of cardiotoxicity associated with sunitinib or sorafenib therapy who receive optimal cardiac treatment survive as long as the patients without any cardiovascular complications.

During the study, aggressive antihypertensive therapy to reach BP goals of $<140 / 90 \mathrm{~mm} \mathrm{Hg}$ (clinic measurements) and $<135 / 85 \mathrm{~mm} \mathrm{Hg}$ (home monitoring) may have reduced the risk of symptomatic heart failure (grade 3 or 4 according to CTCAE) to an insignificant level. Similarly, treating systolic BP and diastolic BP to targets that are $<140$ and $<90 \mathrm{~mm} \mathrm{Hg}$, respectively, is associated with a decrease in cardiovascular complications in the general population of hypertensive patients $[23,24]$. Thus, it is important for oncologists to focus on establishing optimal antihypertensive treatment in these patients and reserving dose manipulations for instances of severe hypertension that cannot be controlled by medical management. Further, our analysis suggests that patients with controlled hypertension should be further evaluated in prospective clinical trials, independent of whether they initially have a grade 2 or grade 3 hypertension according to CTCAE.

Moreover, in our study a substantial proportion of patients in the hypertensive group required more than one antihypertensive medication to lower their BP to the target of $<140 / 90 \mathrm{~mm} \mathrm{Hg}$ (clinic measurements) and $<135 / 85$ $\mathrm{mm} \mathrm{Hg}$ (home monitoring). Indeed, we found that as the number of antihypertensive drugs taken by patients for sunitinib-induced hypertension increased, patient prognosis improved. The need to use several antihypertensive drugs with different mechanisms of action to reach BP targets during this study may be evidence for the physiological complexity of the mechanism by which sunitinib raises $\mathrm{BP}$.

A negative aspect of our findings is the absence of randomization of antihypertensive drugs. Our observations, however, were conducted during the early stages of clinical trials using sunitinib, and, therefore, it was difficult to also consider the randomization of simultaneous antihypertensive treatment. In practice, we concentrated on the patients' clinical situation. Moreover, patients with only radiological disease progression during sunitinib treatment received the next possible drug (sorafenib, everolimus) or were included to other clinical trials. Increasingly, targeted antiangiogenic therapies are effective enough to allow their sequential administration and, thus, groups of patients in whom it is possible to establish the effects of a particular antihypertensive drug on overall mortality are difficult to find. Our observations included a substantial and homogeneous population of patients.

In conclusion, hypertension related to sunitinib was a positive predictive factor associated with significantly longer OS and PFS in patients with $\mathrm{mRCC}$ in this retrospective study. In addition, preexisting optimally treated hypertension was not related to significant worse prognosis in patients with mRCC. Hypertension related to sunitinib treatment often required complex therapy with several antihypertensive medicines, and patients receiving at least three antihypertensive drugs had significantly better prognosis, with longer OS and PFS.

\section{References}

1 Launay-Vacher V, Deray G: Hypertension and proteinuria: a class-effect of antiangiogenic therapies. Anticancer Drugs 2009;20: 81-82.

$\checkmark 2$ Sica DA: Angiogenesis inhibitors and hypertension: an emerging issue. J Clin Oncol 2006;24:1329-1331.

- 3 Shiojima I, Sato K, Izumiya Y, Schiekofer S, Ito M, Liao R, Colucci WS, Walsh K: Disruption of coordinated cardiac hypertrophy and angiogenesis contributes to the transition to heart failure. J Clin Invest 2005;115:21082118.

-4 Hubner N, Yagil C, Yagil Y: Novel integrative approaches to the identification of candidate genes in hypertension. Hypertension 2006; $47: 1-5$.
5 Robinson ES, Khankin EV, Karumanchi SA, Humphreys BD: Hypertension induced by vascular endothelial growth factor signaling pathway inhibition: mechanisms and potential use as a biomarker. Semin Nephrol 2010; 30:591-601.

6 Szmit S, Opolski G, Szczylik C: Powikłania kardiologiczne jako powikłania terapii celowanych. Współczesna Onkol 2008;12:7: 318-323.

7 Szmit S, Nurzynski P, Szalus N, Opolski G, Szczylik C: Reversible myocardial dysfunction in a young woman with metastatic renal cell carcinoma treated with sunitinib. Acta Oncol 2009;4:1-5
8 Zhu X, Stergiopoulos K, Wu S: Risk of hypertension and renal dysfunction with an angiogenesis inhibitor sunitinib: systematic review and meta-analysis. Acta Oncol 2009;48: 9-17.

9 Rini BI, Schiller JH, Fruehauf JP, Cohen EE, Tarazi JC, Rosbrook B, Ricart AD, Olszanski AJ, Kim S, Spano J: Association of diastolic blood pressure $>90 \mathrm{~mm} \mathrm{Hg}$ with overall survival in patients treated with axitinib. J Clin Oncol 2008;26:15S, abstr 3543.

10 Rini BI: Biomarkers: Hypertension following anti-angiogenesis therapy. Clin Adv Hematol Oncol 2010;8:415-416.

11 Force T, Krause DS, Van Etten R: Molecular mechanisms of cardiotoxicity of tyrosine kinase inhibition. Nat Rev Cancer 2007;7:332344. 
12 Motzer RJ, Bacik J, Mazumdar M: Prognostic factors for survival of patients with stage IV renal cell carcinoma: Memorial SloanKettering Cancer experience. Clin Cancer Res 2004;15:6302S-6303S.

13 Therasse P, Arbuck SG, Eisenhauer EA, Wanders J, Kaplan RS, Rubinstein L, Verweij J, Van Glabbeke M, van Oosterom AT, Christian MC, Gwyther SG: New guidelines to evaluate the response to treatment in solid tumors. European Organization for Research and Treatment of Cancer, National Cancer Institute of the United States, National Cancer Institute of Canada. J Natl Cancer Inst 2000;92:205-216.

-14 Di Bari M, Pozzi C, Cavallini MC, Innocenti F, Baldereschi G, De Alfieri W, Antonini E, Pini R, Masotti G, Marchionni N: The diagnosis of heart failure in the community. Comparative validation of four sets of criteria in unselected older adults: the ICARe Dicomano Study. J Am Coll Cardiol 2004;44: 1601-1608.

$\checkmark 15$ European Society of Hypertension-European Society of Cardiology Guidelines Committee: 2003 European Society of HypertensionEuropean Society of Cardiology guidelines for the management of arterial hypertension. J Hypertens 2003;21:1011-1153.
16 O’Brien E, Asmar R, Beilin L, Imai Y, Mallion JM, Mancia G, Mengden T, Myers M, Padfield P, Palatini P, Parati G, Pickering T, Redon J, Staessen J, Stergiou G, Verdecchia P; on behalf of the European Society of Hypertension Working Group on Blood Pressure Monitoring: European Society of Hypertension recommendations for conventional, ambulatory and home blood pressure measurement. J Hypertens 2003;21:821-848.

17 Bono P, Rautiola J, Utriainen T, Joensuu H: Hypertension as predictor of sunitinib treatment outcome in metastatic renal cell carcinoma. Acta Oncol 2011;50:569-573.

-18 Bono P, Elfving H, Utriainen T, Osterlund P, Saarto T, Alanko T, Joensuu H: Hypertension and clinical benefit of bevacizumab in the treatment of advanced renal cell carcinoma. Ann Oncol 2009;20:393-394.

19 Sire M, Wallerand H, Kilkoski F, Grenier N, Ferrière JM, Ravaud A: Anti-angiogenic treatment in the management of metastatic renal cell carcinoma. Bull Cancer 2008;95: 813-820.

20 Weikert S, Boeing H, Pischon T, et al: Blood pressure and risk of renal cell carcinoma in the European prospective investigation into cancer and nutrition. Am J Epidemiol 2008 167:438-446.
21 Di Lorenzo G, Autorino R, Bruni G, et al: Cardiovascular toxicity following sunitinib therapy in metastatic renal cell carcinoma: a multicenter analysis. Ann Oncol 2009;20: 1535-1542.

22 Schmidinger M, Zielinski CC, Vogl UM, Bojic A, Bojic M, Schukro C, Ruhsam M, Hejna $\mathrm{M}$, Schmidinger $\mathrm{H}$ : Cardiac toxicity of sunitinib and sorafenib in patients with metastatic renal cell carcinoma. J Clin Oncol 2008;26:5204-5212.

23 Chobanian AV, Bakris GL, Black HR, et al: Seventh report of the Joint National Committee on Prevention, Detection, Evaluation, and Treatment of High Blood Pressure. Hypertension 2003;42:1206-1252.

24 Mancia G, De Backer G, Dominiczak A, et al: ESH-ESC Task Force on the Management of Arterial Hypertension. J Hypertens 2007;25: 1751-1762. 